\title{
REDUCTION OF THE 1/F NOISE INDUCED PHASE NOISE IN A CMOS RING OSCILLATOR BY INCREASING THE AMPLITUDE OF OSCILLATION
}

\author{
S.L.J. Gierkink ${ }^{\prime \prime}$, A. van der Wel ${ }^{1)}$, G. Hoogzaad ${ }^{2)}$, E.A.M. Klumperink ${ }^{\prime \prime}$, A.J.M. van Tuijl ${ }^{1,3)}$ \\ 1) MESA Research Institute \\ University of Twente \\ 2) Formerly with 1), currently with \\ 3) Philips Semiconductors \\ Philips Research Laboratories \\ Enschede, The Netherlands \\ Eindhoven, The Netherlands \\ Nijmegen, The Netherlands
}

\begin{abstract}
Spectrum measurement results of a CMOS ring oscillator are presented that show a $10 \mathrm{~dB}$ decrease in $1 / \mathrm{f}$ noise induced phase noise at a $2 \mathrm{~dB}$ increase in carrier power. Simple ring oscillator theory predicts that the $1 / \mathrm{f}$ noise induced phase noise is independent of carrier power. It is shown that an increase in the amplitude of oscillation is accompanied by a reduction of the intrinsic $1 / \mathrm{f}$ noise of the periodically switched MOS transistors in the ring. A net reduction of the $1 / \mathrm{f}$ noise of a periodically switched NMOS transistor of more than $12 \mathrm{~dB}$ is measured in baseband.
\end{abstract}

\section{INTRODUCTION}

Ring oscillators are widely used in application areas like video, audio and dataprocessors, where their phase noise parameter critically affects system performance. The impetus of the work presented here was provided by the desire to optimize the jitter performance of a CMOS ring oscillator, to be used in a PLL that generates the pixel clock in a television receiver. Recently, some papers have been presented $[1,2,3]$ that analyze timing jitter of ring oscillators due to only white noise, an approach usually justified by the large loop bandwidth of the PLL. The loop bandwidth of the PLL in our application is only a few $\mathrm{kHz}$ whereas the typical $1 / \mathrm{f}$ corner frequency of the MOS devices exceeds $1 \mathrm{MHz}$. Thus $1 / \mathrm{f}$ noise is expected to play a significant role in the jitter (or phase noise) of the pixel clock.

However, measurement results of a test chip (realized in an industrial $1 \mu \mathrm{m}$ CMOS process) revealed that the $1 / \mathrm{f}$ noise induced phase noise was much smaller than theoretically expected. Since several explanations failed to clear up this discrepancy, a rather bold hypothesis was posed : periodically switching the gate-source voltage of a MOS transistor between an ON- and OFF state affects the physical process that generates $1 / f$ noise, resulting in a reduction of $1 / \mathrm{f}$ noise.

The primary objective of this paper is to show that the $1 / \mathrm{f}$ noise induced phase noise of a CMOS ring oscillator reduces when the amplitude of oscillation is increased. Secondly, measurement results will be presented that support the hypothesis that this reduction can be related to a reduction of the intrinsic $1 / \mathrm{f}$ noise of a periodically switched MOS transistor.

This work was supported by Philips Research Laboratores, Eindhoven and Philips Semiconductors, Nijmegen, The Netherlands.
Recently we found that the effect has been observed before in a weaker form with an alternative experimental setup [4]. However, to our knowledge, we are the first to explore the impact of the effect in a practical application.

The results presented here are relevant for the study of the $1 / \mathrm{f}$ noise induced phase noise in CMOS oscillators. The contribution of $1 / \mathrm{f}$ noise to the phase noise in CMOS oscillator topologies can be lowered significantly by increasing the amplitude of oscillation as this can result in a reduction of the intrinsic 1/f noise of MOS transistors in baseband.

\section{PHASE NOISE MEASUREMENT}

Figure 1 shows the experimental setup. Measurements were carried out on a 3 stage ring oscillator with single ended inverters. For reasons of experimental flexibility and to be able to test devices from different manufacturers, we used the commercially available IHEF4007UB chip containing 3 inverters. The amplitude of oscillation (see figure 2) can be changed significantly by slightly adjusting the value of the resistor $\mathrm{R}_{\mathrm{L}}$.

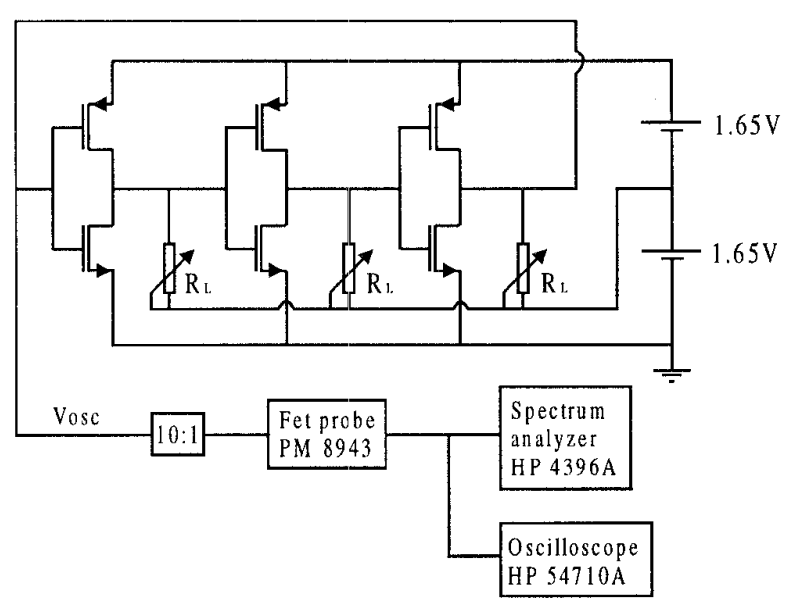

Figure 1. Phase noise measurement setup.

In the following all spectra are single sided. Using the experimental setup of figure 1 , the power spectral density of phase fluctuations can be quantified by first calculating the ratio of the noise power $P_{\text {sideband, } 1 \mathrm{~Hz}}\left(\mathrm{~F}_{\mathrm{m}}\right)$ measured in a sideband of 
the oscillator spectrum over a bandwidth of $1 \mathrm{~Hz}$ at a frequency offset $\mathrm{F}_{\mathrm{m}}$ from the carrier to the carrier power $\mathrm{P}_{\text {canier }}$ :

$$
L\left(F_{m}\right)=\frac{P_{\text {sideband, }, \text { Hz }}\left(F_{m}\right)}{P_{\text {carrier }}}
$$

The inverse of $L\left(F_{m}\right)$ is sometimes denoted as carrier-to-noise ratio. If the phase deviation is much smaller than $1 \mathrm{rad}$ and amplitude modulation is negligible, the phase noise power spectral density $S_{\Delta \phi}$ can be derived from this measure :

$$
S_{\Delta \varphi}\left(F_{m}\right)=2 \cdot L\left(F_{m}\right)
$$

The phase noise is commonly specified as a logarithmic function of $L\left(F_{m}\right)$ :

$$
10 \cdot \log \left(L\left(F_{m}\right)\right) \quad[d B c / H z]
$$

Note that $L\left(F_{m}\right)$ is a relative measure i.e. relative to the carrier power. The carrier power and the sideband power spectral density are measured separately using the spectrum analyzer.

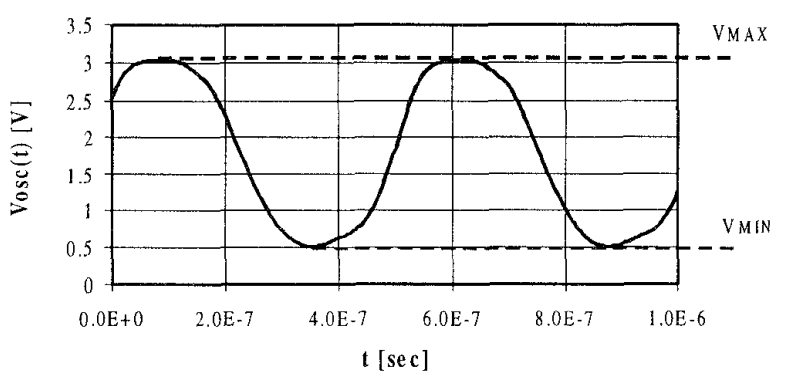

Figure 2. Typical oscillator waveform (unattenuated).

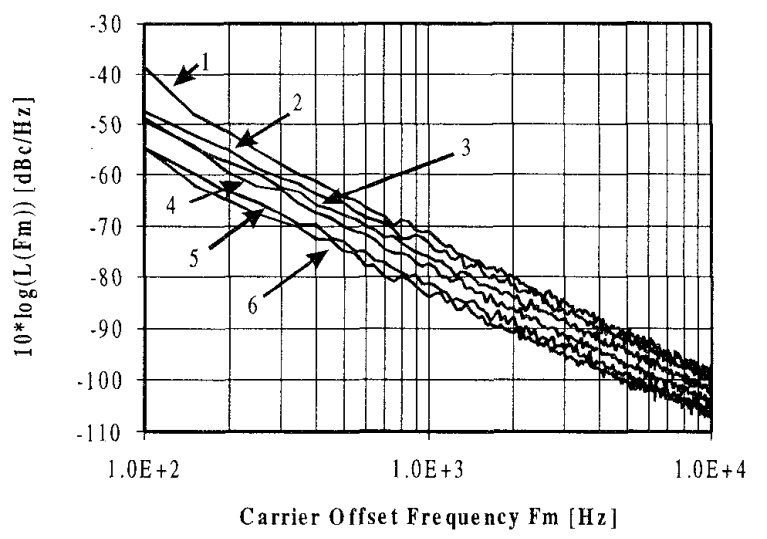

Figure 3 Measured $10 \cdot \log \left(L\left(F_{m}\right)\right)$ as a function of carrier offset frequency at different levels of carrier power (see also table 1).

The oscilloscope is used to determine the maximum and minimum value of the oscillator output voltage waveform (see figure 2). Figure 3 shows the measured $10 \cdot \log \left(\mathrm{L}\left(\mathrm{F}_{\mathrm{m}}\right)\right)$ as a function of carrier offset frequency $\mathrm{F}_{\mathrm{m}}$ at different amplitudes of oscillation. Table 1 shows the corresponding values of $R_{L}$, the frequency of oscillation, the maximum and minimum voltage of the output waveform and the carrier power. A change of $R_{L}$ also results in a small frequency change.

The curves in figure 3 show a sideband slope of about $-25 \mathrm{~dB} / \mathrm{dec}$. In case of ideal $1 / \mathrm{f}$ noise modulating the frequency of oscillation, a sideband slope of $-30 \mathrm{~dB} / \mathrm{dec}$ is theoretically expected. The measured sideband slope is in agreement with the measured slope of the baseband 1/f noise spectrum of switched NMOS transistors. As will be shown in section 4, this slope is slightly less steep than $-10 \mathrm{~dB} / \mathrm{dec}$.

Figure 3 together with table 1 shows that an increase in carrier power of $2 \mathrm{~dB}$ gives rise to a more than $10 \mathrm{~dB}$ decrease in phase noise power spectral density in the $1 / \mathrm{f}$ noise induced part of the sideband spectrum. This is not in good agreement with expectations based on existing oscillator theory, that predicts a $1 / \mathrm{f}$ noise induced phase noise power spectral density that is more or less independent of carrier power, as will be shown in the next section.

\begin{tabular}{|c|c|c|c|c|c|}
\hline $\begin{array}{c}\text { curve } \\
\text { nr. } \\
\text { (Fig.3) }\end{array}$ & $\begin{array}{c}\mathrm{R}_{\mathrm{L}} \\
{[\mathrm{k} \Omega]}\end{array}$ & $\begin{array}{c}\text { FoSC } \\
{[\mathrm{MHz}]}\end{array}$ & $\begin{array}{c}\mathrm{V}_{\mathrm{MAx}} \\
{[\mathrm{V}]} \\
\text { (Fig.2) }\end{array}$ & $\begin{array}{c}\mathrm{V}_{\mathrm{MIN}} * \\
{[\mathrm{~V}]} \\
\text { (Fig.2) }\end{array}$ & $\begin{array}{c}\text { carrier } \\
\text { power* } \\
{[\mathrm{dBV}]}\end{array}$ \\
\hline 1 & 9.4 & 1.702 & 2.98 & 0.59 & -0.68 \\
\hline 2 & 9.5 & 1.772 & 3.01 & 0.58 & -0.60 \\
\hline 3 & 9.6 & 1.773 & 3.02 & 0.54 & -0.36 \\
\hline 4 & 10 & 1.780 & 3.04 & 0.49 & -0.16 \\
\hline 5 & 11 & 1.817 & 3.07 & 0.38 & 0.42 \\
\hline 6 & 15 & 1.867 & 3.25 & 0.23 & 1.32 \\
\hline
\end{tabular}

Table 1.Parameters corresponding with the curves in figure $3(*$ : unattenuated values).

\section{HYPOTHESIS}

The only possible origin of significant $1 / \mathrm{f}$ noise is in the MOS transistors, because the resistors and the batteries used in the experimental setup showed no significant $1 / \mathrm{f}$ noise. According to oscillator theory presented in [3] baseband $1 / \mathrm{f}$ noise can be transferred to the carrier sideband spectrum by means of two mechanisms :

- baseband $1 / \mathrm{f}$ noise is multiplied by the harmonics of the oscillator waveform due to either nonlinearity of the inverter or sampling of the noise by the inverter. A comparison of the maximum and minimum values of the oscillator waveform with the threshold voltages of the MOS transistors indicates that the inverters in the ring experience hard switching. During one period of oscillation, noise present at the input of an inverter is sampled once by the NMOS and once by the PMOS transistor. If there is asymmetry between these two transistors noise at the input of the inverter is multiplied by the fundamental component (carrier). As a result of this 
multiplication by the carrier, the $1 /$ f noise power spectrum is shifted towards the carrier frequency and its magnitude becomes proportional to carrier power. The noise is filtered by the oscillator transfer function (which is assumed to be independent of carrier power) changing the slope of the shifted $1 / \mathrm{f}$ noise by an additional $-20 \mathrm{~dB} / \mathrm{dec}$. The resulting $1 / \mathrm{f}$ noise induced phase noise of the oscillator output signal is independent of the carrier power.

- modulation of the frequency of oscillation due to noise on the inverter output currents. Since the frequency of oscillation is a function of the output current of the inverters, noise on this current modulates the frequency. According to FM modulation theory the baseband 1/f noise is multiplied by the carrier, subsequently filtered with an additional $-20 \mathrm{~dB} / \mathrm{dec}$ (due to the transformation of frequency fluctuations to phase fluctuations) and is finally shifted to the carrier frequency. The resulting $1 / f$ noise induced phase noise of the oscillator output signal is independent of the carrier power.

Assuming that the strength of the $1 / \mathrm{f}$ noise generated in the MOS transistors does not depend on the amplitude of oscillation, the above mentioned mechanisms would give a $1 / \mathrm{f}$ noise induced phase noise which is independent of carrier power. This is not in agreement with our measurements which show that a $2 \mathrm{~dB}$ increase in carrier power leads to a more than $10 \mathrm{~dB}$ decrease in $1 / \mathrm{f}$ noise induced phase noise. In search of an explanation for the effect it is worthwhile to investigate whether the 1/f noise of the MOS transistors in the ring is affected by the amplitude of oscillation. A conclusive approach is then to perform measurements of the $1 / \mathrm{f}$ noise of periodically switched MOS transistors directly in baseband.

In the ring oscillator of figure 1 , the amplitude of oscillation affects both the gate-source and the drain-source voltage of the MOS transistors. From MOS physics it is known that the $1 / \mathrm{f}$ noise output current of a MOS transistor becomes zero when the drain-source voltage reaches zero Volts. This effect should be accounted for when a quantitative explanation is sought for the measurement results of figure 3 . In the next section it is shown that this is not the only effect that plays a role in the relation between the amplitude of oscillation in the ring and the baseband $1 / \mathrm{f}$ noise of the MOS transistors.

\section{BASEBAND MEASUREMENT}

In this section, only the effect of switching of the gate-source voltage of a MOS transistor on its $1 / \mathrm{f}$ noise current in baseband is investigated. To measure the baseband $1 / \mathrm{f}$ noise of MOS transistors whose gate-source voltage is switched, the experimental setup depicted in figure 4 is used. The gates of two NMOS transistors are driven by a square wave with $50 \%$ duty cycle and a maximum voltage level of $V_{G S_{-} O N}$ and a minimum voltage level $\mathrm{V}_{\text {GS }}$ ofF. In this experiment we use NMOS transistors rather than PMOS, since their $1 / \mathrm{f}$ noise corner frequency is much higher. In order to minimize the drainsource voltage fluctuations of the MOS transistors the MOS transistors are cascoded with bipolar transistors. Bipolar transistors have a much lower $1 / \mathrm{f}$ corner frequency than MOS transistors and in cascode configuration their noise contribution is negligible. To ensure that the $1 / \mathrm{f}$ noise of the MOS transistors dominates over the thermal noise of the resistors the product of the transconductance value of the MOS transistors and the resistor value $R_{c}$ is chosen much larger than one. To suppress the large common mode switching signal the output noise voltage is measured using a differential probe. The resistors and capacitors are adjusted to compensate the effect of MOS transistor mismatch.

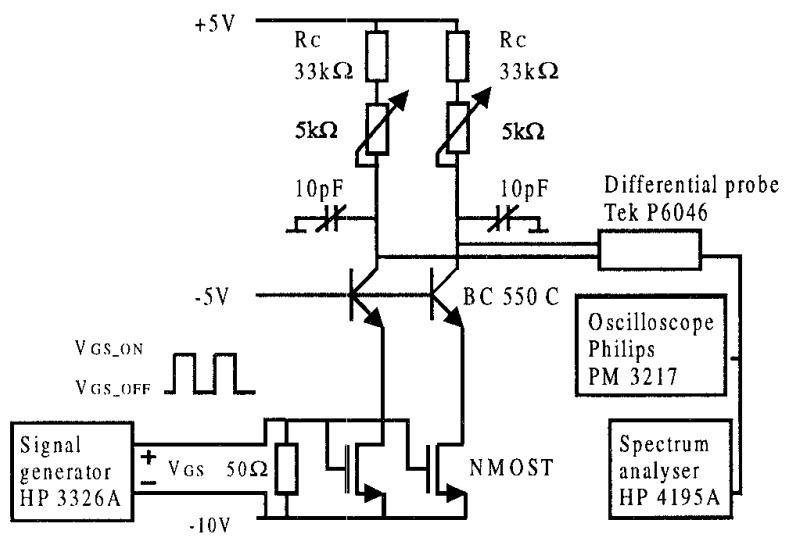

Figure 4 Baseband 1/f noise measurement setup.

Figure 5 shows the measurement results obtained with the setup of figure 4 . It shows the $1 / \mathrm{f}$ noise spectrum for the case of unswitched and switched transistors, the latter at different values of $\mathrm{V}_{\text {GS_OFF }}$ (see table 2 ).

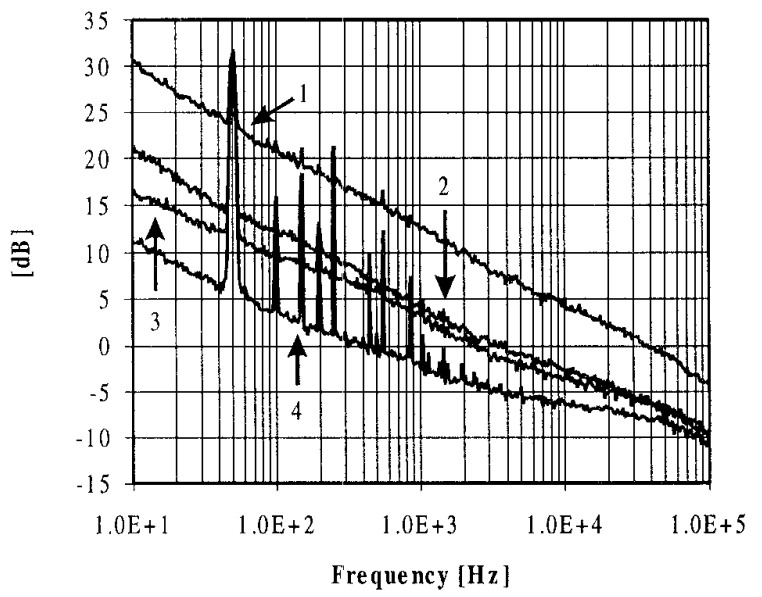

Figure 5 Measured baseband 1/f noise in the unswitched case (curve nr. 1) and switched cases (curves 2 - 4). For $V_{G S}$ values : see table 2 . Switching frequency is $2 \mathrm{MHz}$, duty cycle is $50 \%$.

In all cases the frequency of the square wave is $2 \mathrm{MHz}$ (comparable to the frequency of oscillation of the ring oscillator), its duty cycle is $50 \%$ and the value of $V_{G S O N}$ is 
$2.5 \mathrm{~V}$. No correction for duty cycle has been applied to the spectra shown figure 5 . At a frequency of about $100 \mathrm{kHz}$, the influence of the pole due to the output nodes in the experimental setup becomes noticeable. The distinct spikes in figure 5 are spurious $50 \mathrm{~Hz}$ line harmonics.

\begin{tabular}{|c|c|}
\hline $\begin{array}{c}\text { curve nr. } \\
\text { (Fig. 5) }\end{array}$ & $\begin{array}{c}\text { VGS_OFF } \\
{[\mathrm{V}]}\end{array}$ \\
\hline 1 & unswitched \\
\hline 2 & 0.7 \\
\hline 3 & 0.4 \\
\hline 4 & 0.0 \\
\hline
\end{tabular}

Table 2. $V_{\text {GS_OFF }}$ values corresponding with the curves in figure 5 ( $\mathrm{V}_{G S_{-} O N}=2.5 \mathrm{~V}$ in all cases). The threshold voltage of the used NMOST transistors $: \mathrm{V}_{\mathrm{TN}} \approx 1.9 \mathrm{~V}$.

\section{DISCUSSION}

The baseband measurements show a significant reduction of the 1/f noise of an NMOS transistor when its gate-source voitage is periodically switched between an ON (inversion) and OFF (accumulation) state. The smaller the value of the gate-source voltage in the OFF state (even if it is already well below the threshold voltage), the larger the noise reduction.

Extensive measurements using switching frequencies below the measurement setup's pole frequency showed that this reduction sets in for frequencies directly below the switching frequency and increases down to a frequency that lies approximately 2 decades below the switching frequency. When the $\mathrm{V}_{\text {GS_OFF }}$ value is chosen small enough (e.g. $0 \mathrm{~V}$ ) the noise spectrum becomes flat in this particular frequency region. This can be observed to some extent from curve 4 in figure 5 , although the measurement setup's pole obscures a firm conclusion. For even lower frequencies the noise spectrum takes on a $1 / f$ dependency again, a phenomenon that is also apparent from figure 5 (curves 2-4).

The slope of the baseband $1 / f$ noise spectrum in the unswitched case (curve 1 in figure 5) is somewhat less negative than -10 $\mathrm{dB} / \mathrm{dec}$ in the frequency range $100 \mathrm{~Hz}-10 \mathrm{kHz}$. The results in figure 5 show a maximum net $1 / \mathrm{f}$ noise reduction of more than $12 \mathrm{~dB}$ at $10 \mathrm{~Hz}$ in the periodically switched case for $\mathrm{V}_{\mathrm{GS}} \mathrm{OFF}=0$ $V$. Experiments with PMOS transistors have also shown a significant reduction in $1 / \mathrm{f}$ noise when switched between an $\mathrm{ON}$ and OFF state. Recently we found that a reduction of $1 / \mathrm{f}$ noise in switched MOS transistors has been reported before in a weaker form with an alternative experimental setup [4].

The measurement results in figure 5 contribute to an explanation for the phase noise measurement results of figure 3 : the baseband 1/f noise of the MOS transistors in the ring oscillator is reduced by periodic switching of the gate-source voltage. The reduction is maximal when the minimum gatesource voltage of the transistors in the ring reach zero Volts, which corresponds to maximum carrier power. Assuming that the amount of upconversion due to sampling and the oscillators sensitivity to frequency modulation remain more or less unaffected by increased levels of carrier power, the $1 / \mathrm{f}$ noise induced sideband power spectral density relative to the carrier power will decrease.

The oscillator's sideband slope as observed in the frequency region of figure 3 is hardly affected by the amplitude of oscillation. This is in agreement with the observation from figure 5 that for frequencies lower than approximately 2 decades below the switching frequency ( $2 \mathrm{MHz}$ in figure 5) the baseband noise spectrum of the switched NMOS transistor takes on a $1 / f$ dependency again, independent of the $\mathrm{V}_{\text {GS_OFF }}$ value.

More study is needed to quantify the relation between the decrease in $1 / \mathrm{f}$ noise induced phase noise as a function of the amplitude of oscillation and the decrease in baseband 1/f noise of periodically switched MOS transistors. This is due to the fact that not only the switching of the gate-source voltage but also the switching of the drain-source voltage of the MOS transistors in the ring plays a role in the amount of $1 / f$ noise induced phase noise.

\section{CONCLUSION}

Measurement results are presented that show that the $1 / \mathrm{f}$ noise induced phase noise in a CMOS ring oscillator can be reduced (and carrier power increased) significantly by increasing the amplitude of oscillation. Baseband measurement results show that an increase in the amplitude of oscillation can be accompanied by a reduction of the intrinsic $1 / \mathrm{f}$ noise of a MOS transistor, due to the switching of the gate-source voltage between an ON (inversion) and OFF (accumulation) state. A 10 $\mathrm{dB}$ decrease in $1 / \mathrm{f}$ noise induced phase noise is measured at a 2 $\mathrm{dB}$ increase in carrier power. A net reduction of $12 \mathrm{~dB}$ of the $1 / \mathrm{f}$ noise of a periodically switched NMOS transistor is measured in baseband.

\section{REFERENCES}

[1] J. A. McNeill, "Jitter in Ring Oscillators", IEEE Journal of Solid-State Circuits, Vol. 32, No. 6, pp. 870-879, June 1997.

[2] T.C. Weigandt, B. Kim and P.R. Gray, "Analysis of timing jitter in CMOS ring oscillators", Proc. 1994 ISCAS, May 1994, Vol. 4, pp. 27-30.

[3] B. Razavi, "A Study of Phase Noise in CMOS Oscillators ", IEEE Journal of Solid-State Circuits, Vol. 31, No. 3, pp. 331-343, March 1996.

[4] I. Bloom, Y. Nemirovsky, "1/f Noise Reduction of MetalOxide-Semiconductor Transistors by cycling from inversion to accumulation", Applied Physics Letters, Vol. 58 (15), pp. 1664-1666, April 1991. 